\title{
Mass Gap Problem Physical Solution
}

\author{
Amrit Šorli Srečko \\ Scientific Research Centre Bistra, Slovenia \\ sorli.amrit@gmail.com \\ https://orcid.org/0000-0001-6711-4844
}

\begin{abstract}
A given problem in physics can be solved if it is well formulated. Well formulated means that it has a bijective correspondence to physical reality. Mass Gap Problem has no bijective correspondence with the physical reality and is that's why not solvable mathematically. It can be solved physically by the formulation of the photon's mass accordingly to the Planck-Einstein relation.
\end{abstract}

Keywords: Mass Gap Problem, Planck-Einstein relation, bijectivity.

\section{Introduction}

To solve the Yang-Mills Mass Gap Problem [1] we have to understand well what mass is. I propose a bijectivity solution for the mass-gap problem where every element in the model of physical reality has exactly one correspondent model in physical reality. The model of physical reality is set $\mathrm{Y}$ and physical reality is set $\mathrm{X}$. Every element in set $\mathrm{Y}$ has exactly one element in the set X. For example, element mass $m_{x}$ in the physical universe has correspondent element mass $m_{y}$ in the model of the universe. They are related by the bijective function of set theory:

$$
f: m_{x} \rightarrow m_{y}
$$

Einstein formula $E=m c^{2}$ has bijective correspondence with the physical universe:

$$
\begin{aligned}
& E_{x}=m_{x} c_{x}^{2} \\
& E_{Y}=m_{y} c_{y}^{2}
\end{aligned}
$$

Every object with mass $m$ is existing in space we call today a superfluid quantum vacuum. Space is not "empty", space is energy in the same way as mass is energy [2]. The idea of "empty" space as something real is harming physics for more than 100 years. It is time we 
demolish the idea of empty space. Mass $m$ is in physics an element with the attribute of energy $E$. It is false to think that this element mass can exist in space that has no attribute of energy $E$. The formula (3) below is false:

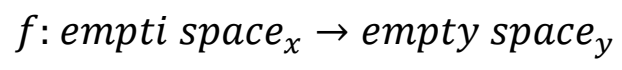

Mass $m$ of a given physical object is related to the energy of space accordingly to the wellknown physical law of homogenous distribution of energy. Every physical system tends that the energy of the system is distributed in a homogeneous way. Every physical object with mass $m$ is diminishing the energy density of space exactly for the amount of its energy $E$ :

$$
\frac{E}{c^{2}}=m=\frac{\left(\rho_{E \max }-\rho_{E \min }\right) V}{c^{2}} \quad \text { (4) }[2]
$$

where $p_{E \max }$ is the energy density of the space infinitely far away from the physical object surface, $p_{E \min }$ is the energy density of the space in the centre of a given physical object and $V$ is the volume of the object. In the Newtonian perspective, the area of space with a higher density is asserting a given pressure towards the area of space with a lower density. From a quantum physics perspective, universal space has the vector orientation towards lower energy density, or in space are quantum fluctuations towards lover energy density of space.

The variable energy density of space is giving the origin to the inertial mass $m_{i}$ and gravitational mass $m_{g}$ as follows in Eq. (5) below:

$$
m_{i}=m_{g}=\frac{\left(\rho_{E \max }-\rho_{E \min }\right) V}{c^{2}}
$$

where $\rho_{E \max }$ is the energy density of the space in interstellar space; $\rho_{E \min }$ is the energy density of the space in the centre of the proton, and $V$ is the volume of the proton. 


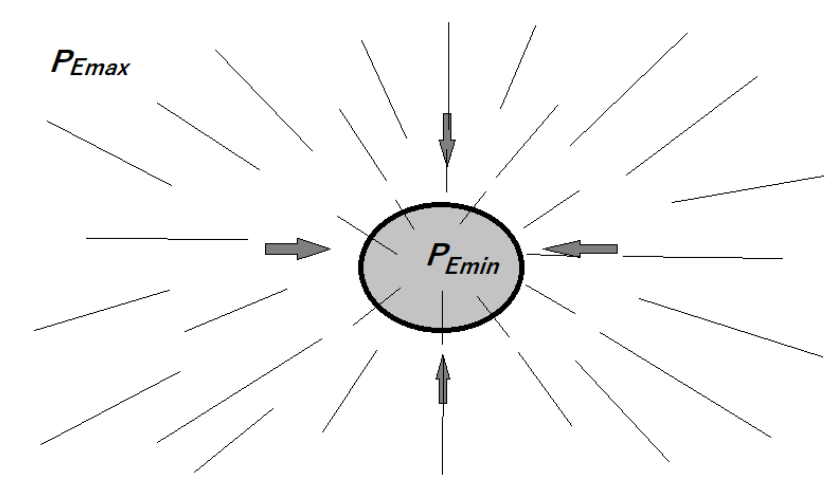

Figure 1: Proton inertial mass and proton gravitational mass have the same origin in variable energy density of space

Formula (5) shows that inertia and gravity are the results of the dynamics between given mass $m$ and a variable energy density of space. It is false to think that inertial mass $m_{i}$ is the same phenomenon as the mass $m$ as the amount of energy $E$. Formula (6) below is false:

$$
\frac{E}{c^{2}}=m=m_{i}
$$

\section{Physical Origin of the Planck Constant}

We can combine equation $E=m c^{2}$ and equation $E=h v$ and we get:

$$
\begin{gathered}
E=m \cdot c^{2}=h \cdot v \\
m=\frac{h \cdot v}{c^{2}}
\end{gathered}
$$

Photon is massless in the sense that it has no inertial mass $m_{i}$. But photon has energy $E$ and so it has correspondent mass $m$. Formula (8) is showing the mass of the photon related to its frequency.

Combining (4) and (8) we get:

$$
m=\frac{\left(\rho_{E \max }-\rho_{E \min }\right) V}{c^{2}}=\frac{h v}{c^{2}}
$$

Out of (9) follows: 


$$
h=\frac{\left(p_{E \max }-p_{E \min }\right) V}{v}
$$

The space pressure $\rho_{E \max }-\rho_{E \min }$ we can express as $\Delta_{E \rho}=\rho_{E \max }-\rho_{E \min }$ and we get:

$$
h=\frac{\Delta_{E \rho} V}{v}
$$

Eq. (11) shows the relation between the space pressure $\Delta_{E \rho}$, volume $\mathrm{V}$ of a given particle and frequency $v$ of a given particle is constant, we call it Planck constant $h$.

\section{Physical Aspects of Mas Gap Problem}

For Yang-Mills Mass Gap Solution is required: "It must have a "mass gap;" namely there must be some constant $\Delta>0$ such that every excitation of the space has energy at least $\Delta$ “ [1]. Eq. (11) shows this constant $\Delta>0$ is Planck constant $h$. Namely, every particle can be seen as the excitation of the space and is defined by the difference of vacuum pressure $\Delta p_{E}$, volume $\mathrm{V}$ and frequency $v$ of a given particle. These three elements are defining the Planck constant $h$. A given excitation of space is a given elementary particle with energy $E$. We know the origin of the Planck constant (Eq. 11) and we see that the minimal "mass gap" in the excitation of space is defined by the Planck constant $h$. In physical terms "mass gap" would be better named as "mass-energy gap", because Einstein told us already that mass and energy are made out of the same "stuff". What's this "stuff" is being clearly explained by Erving Schrodinger who also regarded space as the fundamental energy of the universe: "What we observe as material bodies and forces are nothing but shapes and variations in the structure of space“.

In the Bohr model of the Hydrogen atom, it is supposed that the photon is released when an electron jumps from higher on a lower orbit, see figure below: 


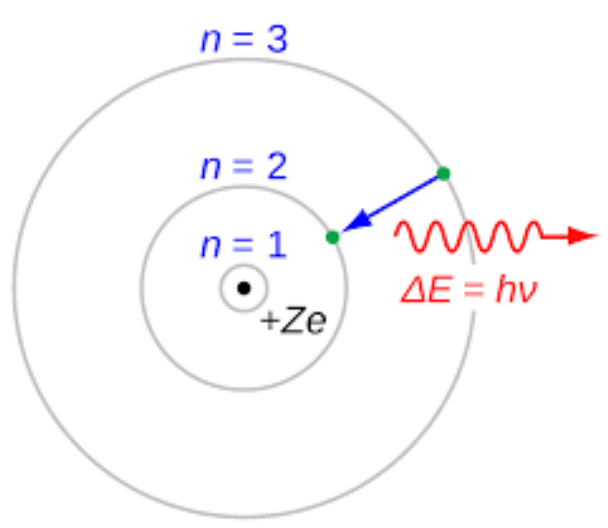

Figure 2: Bohr Hydrogen atom

We will perform a simple experiment. We close the hydrogen gas in a glass chamber in and we heat the chamber. The gas will heat, the electrons will jump on higher orbits and gas will emanate photons. From where are coming these photons? Are they hidden somewhere on the atomic structure of the Hydrogen? They are not hidden in the atomic structure; they are hidden in the space [3]. By heating gas, we are also heating the vacuum, because hydrogen atoms exist in the vacuum. In the heated vacuum (space) virtual photons become visible, they emanate from the gas. When we stop heating the glass chamber the hydrogen gas too will start cooling. Electrons will turn from higher to the lower orbits and gas when cooling down will stop emanating photons because the space (superfluid quantum vacuum) will also cool down. The fact is, the hydrogen atom and in generally every atom is the energy structure of the space. We should not see atom as an isolated structure apart from the space.

Max Planck formulated the quantum theory by saying that light that was emitted had discrete levels of energy, and that energy that was radiated was quantized:

$$
E=n h v \quad(12)
$$

where $n=1,2,3 \ldots$ is integer number, $h$ is Planck constant and $v$ is the photon frequency. Quantization of energy states that there are discrete values or states, and energies in between the values of $\mathrm{n}$ are forbidden. The minimal change of the radiation is related to the Planck constant $h$.

Computer simulations suggest the existence of a "mass gap" in the solution to the quantum versions of the Yang-Mills equations. But no mathematical proof of this property is known [1]. My intent in this article is to find the solution for the "Yang-Mills Mass Gap problem" from the perspective of physics by using bijectivity. Euclidean Green's functions are 
abstract mathematical objects which do not have bijective correspondence with the physical world. Osterwalder and Schrader are introducing Euclidean vector $\mathbb{R}^{4}$ with "time direction" that is orthogonal to the "space directions" [4]. With Fiscaletti and Čelan we proved time is not the 4th dimension of the universal space and has no direction. There is no such thing in the universe as "time direction". Universal space is time-invariant. In the universal space, time is the duration of change, i.e., motion. Duration, in order to exist, has to be measured from the side of the observer $[5,6]$.

Einstein has interpreted the time $t$ as the $4^{\text {th }}$ coordinate $X_{4}$ of a Minkowski manifold. He wrote: "If we replace $x, y, z, \sqrt{-1} c t$ by $x_{1}, x_{2}, x_{3}, x_{4}$, we also obtain the result that $d s^{2}=d x_{1}^{2}+$ $d x_{2}^{2}+d x_{3}^{2}+d x_{4}^{2}$ is independent of the choice of the body of reference. We call the magnitude $d s$ the "distance" apart of two events or four-dimensional points. Thus, if we choose as time variable the imaginary variable $\sqrt{-1} c t$ instead of the real quantity $t$, we can regard the continuum space-time, in accordance with the special theory of relativity, as an "Euclidean" four-dimensional continuum, a result following by the consideration of the preceding section" [7]. In the above citation, Einstein suggestion that we can choose the time variable $t$ as the imaginary variable can be written as follows:

$$
t=\sqrt{-1} c t
$$

Eq. (13) is false because on the left side of the equation we have $t$ and on the left side we have $\sqrt{-1} c t$. Combining Eq. (6) with equation well know equation $X_{4}=i c t$ we get:

$$
X_{4}=i t c^{2} \sqrt{-1}
$$

Einstein did a mistake keeping and interpreting time as the dimension of a four-dimensional continuum. Physics is still today suffering this misinterpretation of time. There is no time and there is no "time direction" in the universe; this means Osterwalder's and Schrader's mathematical model on Euclidean Green functions [4] has no bijective correspondence with the physical world and cannot contribute to the solution of the "Mass Gap". Osterwalder's and Schrader's model has internal inconsistency because it considers the time is a direction. I have to point out here that every model of physical reality which is based on bijectivity has no "internal inconsistencies" and so no "problems". The physical solution "mass gap" problem is that minimal change of "mass-energy" excitation of the space is defined by Planck constant $h$. 


\section{Conclusions}

My proposal for the physical solution of the "mass gap" problem is in accordance with the Planck-Einstein relation. The mathematical solution of the "Yang-Mills Mass Gap Problem" will not be found because Euclidean green functions do not have bijective correspondence with physical reality. "Yang-Mills Mass Gap Problem" is of a mathematical character and is the result of missing bijectivity research methodology in physics. Once we apply bijectivity in physics, where every element of a given equation will have a bijective element in the physical world such problems will not appear anymore.

\section{References:}

1. Clay Mathematics Institute https://www.claymath.org/millennium-problems (2020)

2. Fiscaletti, D., \& Sorli, A. (2018). Quantum Relativity: Variable Energy Density of Quantum Vacuum as the Origin of Mass, Gravity and the Quantum Behaviour. Ukrainian Journal of Physics, 63(7), 623. https://doi.org/10.15407/ujpe63.7.623

3. Fiscaletti, D., \& Sorli, A. (2020). A Three-Dimensional Non-Local Quantum Vacuum as the Origin of Photons. Ukrainian Journal of Physics, 65(2), 106. https://doi.org/10.15407/ujpe65.2.106

4. Osterwalder, K., Schrader, R. Axioms for Euclidean Green's functions. Commun.Math. Phys. 31, 83-112, pp. 85 (1973). https://doi.org/10.1007/BF01645738.

5. Šorli, A., \& Čelan, Štefan. (2020). The End of Space-time: PhysicsMathematics. International Journal of Fundamental Physical Science, 10(4), 31-34. https://doi.org/10.14331/ijfps.2020.330139

6. Fiscaletti, D., Sorli, A. Perspectives of the Numerical Order of Material Changes in Timeless Approaches in Physics. Found Phys 45, 105-133 (2015). https://doi.org/10.1007/s10701-014-9840-y

7. Einstein, A., Relativity: The Special and General Theory, Methuen \& Co Ltd, p.93 (1916). 